\title{
Functional Improvement after Sub-Acromial Corticosteroid Injection in Patient with Shoulder Impingement Syndrome
}

\author{
Khalid Karim, ${ }^{1}$ Ranjit Kumar Sah, ${ }^{2}$ Abdul Rasheed, ${ }^{3}$ Syed Muhammad Awais ${ }^{4}$
}

\begin{abstract}
Background: Sub-acromial impingement syndrome (SAIS) is the most common disorder of shoulder. Conservative treatment is the first choice, often with corticosteroid injection or different physiotherapy interventions, or both. So we aimed to assess the mean reduction in shoulder pain and disability index (SPADI) score after administration of sub-acromial corticosteroid injections for management of SAIS.
\end{abstract}

Objectives: To determine the mean reduction in SPADI score after administration of Sub-acromial cortico-

Date of Submission 10-11-2015

Date of Revision Received 19-1-2016

Date of Acceptance for Publication 13-4-2016

Conflict of Interest: None

Funding Source: None

Karim K. ${ }^{1}$

Resident, Department of Orthopaedic Surgery and Traumatology Unit - I, KEMU/Mayo Hospital, Lahore

Sah R.K. ${ }^{2}$

Resident, Department of Orthopaedic Surgery and

Traumatology Unit - I, KEMU/ Mayo Hospital, Lahore

Rasheed A. ${ }^{3}$

Resident, Department of Orthopaedic Surgery and Traumatology Unit - I, KEMU/ Mayo Hospital, Lahore

Awais S.M. ${ }^{4}$

Chairman, Department of Orthopaedic Surgery and Traumatology, KEMU / Mayo Hospital, Lahore steroid injections for management of sub-acromial impingement syndrome.

Material and Methods: The Quasi - experimental study was conducted in Department of Orthopedic Surgery, Mayo Hospital Lahore from November 2013 to august 2014. 150 cases were enrolled in this study. Then baseline SPADI score was noted. Patients were injected with sub-acromial injections of $40 \mathrm{mg}$ triamcinolone acetonide mixed with $4.5 \mathrm{ml}$ of $2 \%$ lignocaine under aseptic measures. All patients did exercises, consisting of pendulum and range of motion under care of physiotherapist. Each patient was followed-up in OPD for assessment of shoulder function according to SPADI score at 1, 3 and 6 weeks after administration of injection. The result was analyzed by using SPSS version 16.

Results: The mean age of the patients was $44.58 \pm$ 11.48 years. There were $46.67 \%$ males and $53.33 \%$ females in this study. The mean SPADI score of the patients at baseline was noted as $75.94 \pm 7.73$ which was reduced to $46.25 \pm 5.62$ after 6 weeks.

Conclusion: Our study results concluded that Subacromial corticosteroid injection is effective in treatment of shoulder impingement syndrome.

Keywords: SPADI, Corticosteroid Injection, Conservative Management, Sub acromial Impingement syndrome, SIAS, Pain.

\section{Introduction}

Sub-acromial impingement syndrome (SAIS) accounts for $44-65 \%$ of shoulder pain and is the most common disorder of shoulder. ${ }^{1}$ SAIS represents spectrum of disease process that ranges from sub-acromial bursitis to rotator cuff tendinopathy and full thickness tears. ${ }^{2}$ 
Functional loss and disability are the major consequences of SAIS. ${ }^{1}$ This is associated with substantial impact daily activities and on sleeping. ${ }^{3}$

The pathogenesis of rotator cuff degeneration is unknown. Theories for pathogenesis are extrinsic cause i.e. compression of the rotator cuff and intrinsic cause i.e. tendon degeneration.

It is diagnosed clinically based on history and clinical signs as Neer impingement sign and Hawkins Kennedy impingement sign. Imaging is helpful adjunct for confirming the diagnosis. MRI is the most helpful technique in evaluating shoulder pain secondary to sub-acromial impingement and rotator cuff disease. ${ }^{4}$

Conservative management with corticosteroid injection and/or physiotherapy is the first choice for SAIS. ${ }^{5}$ Nonsurgical treatment is successful in most patients with SAIS. Surgical intervention is successful in patients in whom nonsurgical treatment fails. ${ }^{1,6}$

Mechanism of action of local steroid injection is not well known. Most likely mechanisms include antiinflammatory effects, influence of local tissue metabolism, pain relief, mechanical improvement, relief of reflex muscle spasm and placebo effect. ${ }^{7}$

Rationale of this study was to determine the mean reduction in SPADI score after administration of subacromial corticosteroid injections for management of SAIS because of unavailability of expertise in our country and absence of local study.

\section{Patient and Methodology}

150 patients presenting to outdoor of Mayo hospital with age greater than 18 years and positive for signs of shoulder impingement syndrome on clinical examination (like Neer impingement test, Hawkins - Kennedy impingement sign, Jobes supraspinatus test) were involved in this study. All the patient with history of shoulder fracture or fracture in the shoulder joint and those with pain referring from cervical spine and those with inflammatory arthritis were excluded from the study. Baseline SPADI score was noted. Patients were injected with sub acromial injections of $40 \mathrm{mg}$ triamcinolone acetonide mixed with $4.5 \mathrm{ml}$ of $2 \%$ lignocaine under aseptic measures. The needle was angled approximately $45^{\circ}$ cephalad to follow the contour of posterior acromion. After injection patients were advised to refrain from heavy arm activities for next 2 weeks. All patients underwent physiotherapy consisting of pendulum and range of motion under care of physiotherapist. Each patient was followed-up in OPD for assessment of shoulder function according to SPADI score at 1, 3 and 6 weeks after administration of injection. Mean of these 3 readings were calculated and subtracted from baseline SPADI score. The data analysis was entered and analyzed by using SPSS version 16 .

\section{Results}

Total 150 patients were enrolled in this study. The mean age of the patients was noted as $44.58 \pm 11.48$ years (range $25-70$ years). $46.67 \%$ patients were males whereas $53.33 \%$ patients were females. The mean SPADI score of the patients at baseline was noted as $75.94 \pm 7.73$ (ranges $60.76-89.23$ ) No drop out was found.

The mean SPADI score of the patients at week 1 was noted as $62.50 \pm 7.58$ (ranges $52.30-75.38$ ).

The mean SPADI score of the patients at week 3 was noted as $52.58 \pm 5.93$ (ranges 44.16 and 61.53).

The mean SPADI score at week 6 of the patients was noted as $46.25 \pm 5.62$ with minimum and maximum values of 36.92 and 56.15 respectively.

In this study the mean SPADI reduction of the patients was noted as $22.15 \pm 3.93$ with minimum and maximum values of 14.35 and 30.77 respectively.

Statistically significant difference was found between the pre SPADI score values comparison with post values at week 1 , week 3 and Week 6 respectively. i.e. $\mathrm{p}$-value $=0.000,0.000,0.000$.

\section{Discussion}

The shoulder impingement syndrome leads to painful shoulder joint along with range of motion limitation and reduction in quality of life. Thirty to $80 \%$ of subacromial injections reaches the sub-acromial bursa or the sub-acromial space when placed blindly. ${ }^{8}$

Our study results showed that the mean SPADI score at baseline was $75.94 \pm 7.73$ and it reduced to $22.15 \pm 3.93$ after the administration of injection. One previous study reported that in patients with sub-acromial impingement syndrome, the baseline mean SPADI score was $46.25 \pm 7.86$ which was reduced to 32.01 \pm 6.76 after 6 weeks of administration of sub-acromial corticosteroid injections. ${ }^{9}$

Our study results showed statistically significance difference between the baseline SPADI score values and follow-up values after administration of sub-acromial corticosteroid injections for management of subacromial impingement syndrome. Many of studies 
support the results in favor of our study. There are studies that reports good short term improvement after accurate placement of injection in the sub-acromial bursa. $^{8,10}$

In study by Niamh Hambly et al they concluded that sub-acromial steroid is helpful for short - term management of clinically and magnetic resonance imaging proven SAIS. Here $83 \%$ patients had relief on $6^{\text {th }}$ month follow-up. It was found that better outcome is in those who have shorter duration of symptoms and minor changes in MRI. ${ }^{4}$

OM Ekeberg et al, found that the mean difference in SPADI from baseline to the 6 week follow-up was 24.4 for those receiving sub-acromial steroid injection and 19.2 for those receiving systemic corticosteroid. The results at 6 week follow-up favored the group having local injections. ${ }^{7}$

In one study it was seen that only $29 \%$ of blindly administered sub-acromial injections are accurately placed. ${ }^{11}$ In a study by Chard et al., $39.4 \%$ of conservatively treated patients reports relief from symptoms at follow-up. ${ }^{12}$

Ali Yeganeh et al, demonstrated in their study that sub-acromial corticosteroid injection results in improvement in the symptoms but long-term results were in favor of physiotherapy. This favors conservative treatment before surgery in patient with sub-acromial impingement syndrome. ${ }^{6}$

Ludewig and Borstad studied the result after home - based physiotherapy for 10 weeks. They established significant improvements in work - related pain and disability, and the shoulder rating questionnaire in the exercise group $(n=34)$ after 10 weeks when compared to a control group $(\mathrm{n}=33)$ receiving no treatment. $^{12}$

Naredo et al and Chen et al, reports ultrasound guided sub-acromial steroid injection to be better than blind injection in SAIS. ${ }^{13}$

Inconclusive results are there regarding the use of local steroid injection in sub-acromial impingement syndrome. Winters et al. categorized patients with shoulder pain clinically into a shoulder girdle and a synovial group. Manipulation is the best treatment for shoulder girdle group, whereas corticosteroid injection is best for synovial group. ${ }^{14}$

\section{Conclusion}

Our study results concluded that sub-acromial corticosteroid injection is an effective drug used for the man- agement of sub-acromial impingement syndrome. However its long term effect needs further follow-up.

\section{References}

1. Umer M, Qadir I, Azam M. Subacromial impingement syndrome. Orthopedic reviews, 2012; 4 (2).

2. Harrison AK, Flatow EL. Subacromial impingement syndrome. Journal of the American academy of Orthopaedic Surgeons, 2011; 19 (11): 701-8.

3. Bennell K, Wee E, Coburn S, Green S, Harris A, Staples M, et al. Efficacy of standardised manual therapy and home exercise programme for chronic rotator cuff disease: randomised placebo controlled trial. Bmj. 2010; 340 .

4. Hambly N, Fitzpatrick P, MacMahon P, Eustace S. Rotator cuff impingement: correlation between findings on MRI and outcome after fluoroscopically guided subacromial bursography and steroid injection. American Journal of Roentgenology, 2007; 189 (5): 1179-84.

5. Holmgren T, Björnsson Hallgren H, Öberg B, Adolfsson L, Johansson K. Effect of specific exercise strategy on need for surgery in patients with subacromial impingement syndrome: randomised controlled study. Bmj. $2012 ; 344$.

6. Yeganeh A, Abdollahi M, Nakhaei Amroodi M, Farahini H. Comparison of the efficacy of local corticosteroid injection and physical therapy on pain severity, joint range of motion and muscle strength in patients with shoulder impingement syndrome referred to Rasool-e-Akram Medical Center from April 2008 to September 2009. Medical Journal of The Islamic Republic of Iran (MJIRI), 2011; 25 (3): 142-52.

7. Ekeberg OM, Bautz - Holter E, Tveitå EK, Juel NG, Kvalheim S, Brox JI. Subacromial ultrasound guided or systemic steroid injection for rotator cuff disease: randomised double blind study. Bmj. 2009; 338.

8. Gruson KI, Ruchelsman DE, Zuckerman JD. Subacromial corticosteroid injections. Journal of Shoulder and Elbow Surgery, 2008; 17 (1): S118-S30.

9. Crawshaw DP, Helliwell PS, Hensor E, Hay EM, Aldous SJ, Conaghan PG. Exercise therapy after corticosteroid injection for moderate to severe shoulder pain: large pragmatic randomised trial. Bmj. 2010; 340.

10. Hollingworth GR, Ellis RM, Hattersley TS. Comparison of injection techniques for shoulder pain: results of a double blind, randomised study. British medical journal (Clinical research ed), 1983; 287 (6402): 1339.

11. Eustace J, Brophy D, Gibney R, Bresnihan B, FitzGerald O. Comparison of the accuracy of steroid placement with clinical outcome in patients with shoulder symptoms. Annals of the rheumatic diseases, 1997; 56 (1): 59-63.

12. Chard MD, Sattell LM, Hazleman BL. The long term outcome of rotator cuff tendonitis: a review study. Br J 
Rheumatol. 1988; 27 (5): 385-9.

13. Ludewig P, Borstad J. Effects of a home exercise programme on shoulder pain and functional status in construction workers. Occupational and environmental medicine, 2003; 60 (11): 841-9.

14. Naredo E, Cabero F, Beneyto P, Cruz A, Mondéjar B, Uson $\mathrm{J}$, et al. A randomized comparative study of short term response to blind injection versus sonographic guided injection of local corticosteroids in patients with painful shoulder. The Journal of rheumatology, 2004; 31 (2): 308-14.
15. Winters JC, Sobel JS, Groenier KH, Arendzen HJ, Jong BM-d. Comparison of physiotherapy, manipulation, and corticosteroid injection for treating shoulder complaints in general practice: randomised, single blind study. Bmj. 1997; 314 (7090): 1320.

\section{Remarks}

Revision with omission of unnecessary statement with correction in grammatical error has been done. 